\title{
MÉTODO CANGURU NA PERSPECTIVA DOS PROFISSIONAIS DE SAÚDE DE UMA UNIDADE DE NEONATOLOGIA
}

Josélia Rodrigues de Souza; Laiane Medeiros Ribeiroli; Géssica Borges Vieira; Laise Escalianti Del Alamo Guardal; Casandra Genoveva Rosales Martins Ponce de Leon'; Juliana Machado Schardosim

Objetivo: analisar a percepção do Método Canguru pelos profissionais de saúde de uma unidade de neonatologia. Metodologia: estudo descritivo, com abordagem qualitativa, realizado por meio de entrevista com roteiro parcialmente estruturado com 19 profissionais de saúde de uma unidade de neonatologia de um hospital público do Distrito Federal, entre abril e maio de 2017. O conteúdo foi analisado segundo a proposta de Bardin e utilizado o software Interface de R pour les Analyses Multidimensionnelles de Textes et de Questionnaires (IRAMUTEQ). A pesquisa foi aprovada pelo Comitê de Ética em Pesquisa. Resultados: os profissionais destacaram a melhora e evolução do quadro clínico do recém-nascido após o contato pele a pele. O conhecimento da equipe em relação ao Método Canguru foi considerado alinhado com a literatura científica. Conclusão: a participação dos pais e irmãos, e o déficit de recursos humanos foram reconhecidos como lacunas para melhor adesão ao método.

Descritores: Método Canguru, Pessoal de Saúde, Enfermagem Neonatal.

\section{KOREAN METHOD IN THE PERSPECTIVE OF THE HEALTH PROFESSIONALS OF A NEONATOLOGY UNIT}

Objective: to analyze the perception of the Kangaroo Method by the health professionals of a Neonatology Unit. Methodology: descriptive study with a qualitative approach, conducted through interview with a partially structured script with 19 health professionals from a Neonatology Unit of a public hospital in the Federal District between April and May 2017. The content was analyzed according to the proposal of Bardin and the Interface Software for Multidimensional Analysis of Texts and Questionnaires (IRAMUTEQ). The research was approved by the Research Ethics Committee. Results: the professionals highlighted the improvement and evolution of the clinical condition of newborns after skin-to-skin contact. The team's knowledge regarding the Kangaroo Method was considered in line with the scientific literature. Conclusion: The participation of parents and brothers along with the shortage of human resources were recognized as gaps for better adherence to the method.

Descriptors: Kangaroo Method, Health Personnel, Neonatal Nursing.

\section{MÉTODO CANGURU EN LA PERSPECTIVA DE LOS PROFESIONALES DE SALUD DE UNA UNIDAD DE NEONATOLOGÍA}

Objetivo: analizar la percepción del Método Canguro por los profesionales de salud de una Unidad de Neonatología. Metodología: estudio descriptivo, con enfoque cualitativo, realizado por medio de entrevista con guión parcialmente estructurado con 19 profesionales de salud de una Unidad de Neonatología de un hospital público del Distrito Federal, entre abril y mayo de 2017. El contenido fue analizado según la propuesta de Bardin y se utilizó el software Interfaz de R para los Analyses Multidimensionnelles de Textes et de Questionnaires (IRAMUTEQ). La investigación fue aprobada por el Comité de Ética en Investigación. Resultados: los profesionales destacaron la mejora y evolución del cuadro clínico del recién nacido después del contacto piel con piel. El conocimiento del equipo con respecto al Método Canguru se consideró en línea con la literatura científica. Conclusión: La participación de padres y hermanos junto con la escasez de recursos humanos se reconocieron como brechas para una mejor adherencia al método.

Descriptores: Método Canguro, Personal de Salud, Enfermería Neonatal. 


\section{INTRODUÇÃO}

O nascimento prematuro resulta na separação precoce entre mãe e bebê devido à necessidade de internação nas Unidades de Terapia Intensiva Neonatal (UTIN) e Unidade de Cuidados Intermediários Neonatal (UCIN). Além disso, a internação de um recém-nascido (RN) em uma UTIN é considerada, pelas mães, um evento devastador e traumático, além de alterar a dinâmica e rotina familiar ${ }^{(1)}$.

Dentre as estratégias para promoção do vínculo mãe e bebê, destaca-se o Método Canguru (MC), que está associado a uma redução da morbimortalidade neonatal, da hipotermia, septicemia, hipoglicemia e readmissão hospitalar. Além disso, há relatos de melhora no humor, proximidade com a criança, aumento do senso de competência no cuidado dos filhos, menos estresse, ansiedade e fadiga ${ }^{(2)}$.

Embora seja uma medida de baixo custo, segura e eficaz, ainda existem barreiras em sua implementação, sendo os profissionais de saúde essenciais para superação das limitações promovidas pelos familiares e demais profissionais( ${ }^{(3,4)}$.

A escolha dessa temática justifica-se por este método ser importante para aumentar o vínculo entre mãe e bebê, incentivando o aleitamento materno e reduzindo o tempo de internação. É necessário acolher a família e dar orientação adequada, portanto optou-se por entrevistar os profissionais de saúde por estarem em contato direto com a família. Pretende-se com este trabalho estimular ainda mais a reflexão e a ação do método canguru nas unidades.

Assim o objetivo foi analisar a percepção do MC pelos profissionais de saúde em uma unidade de neonatologia.

\section{METODOLOGIA}

\section{Tipo de estudo}

Trata-se de uma pesquisa descritivo, com abordagem qualitativa.

\section{Participantes da pesquisa}

A amostra de conveniência foi de 19 profissionais de saúde, sendo: seis técnicos de enfermagem, três enfermeiros, quatro médicos, três fisioterapeutas, duas fonoaudiólogas e uma terapeuta ocupacional que integravam a equipe multiprofissional. Os critérios de inclusão foram: ser profissional na unidade e com experiência de no mínimo seis meses no setor. Não participaram da pesquisa aqueles que estavam de férias ou de licença durante a realização das entrevistas. Os profissionais foram codificados da seguinte forma: $E l$ a E19, sendo que E se refere a Entrevistado e os números arábicos de 1 a 19 se referem à ordem das entrevistas.

\section{Local do estudo}

O estudo desenvolveu-se em uma unidade de neonatologia (UTIN e UCIN) de um hospital público do Distrito Federal, no período de abril a maio de 2017.

\section{Coleta dos dados}

A coleta das informações foi mediante entrevistas com roteiro semiestruturado, gravadas em aparelho digital e transcritas na integra. As entrevistas ocorreram no local e turno de trabalho dos profissionais de saúde, com agendamento prévio, em ambiente reservado, com duração média de 25 minutos. A entrevistadora foi uma acadêmica de enfermagem previamente treinada.

O roteiro contemplava as seguintes questões: 1.Como está sendo utilizado o MC nesta unidade de neonatologia?; 2.Qual o papel que sua categoria profissional desempenha para a realização do MC?; 3. Qual a sua participação na recepção da família?; 4.Qual a importância da participação dos pais e irmãos no método?; 5.Como a família pode contribuir para a recuperação do RN?; 6.Cite um relato da sua assistência que marcou sua vida profissional e/ou pessoal.

\section{Procedimentos de análise dos dados}

Para análise dos dados, foi realizada a análise de conteúdo proposta por Bardin ${ }^{(5)}$ e utilizado o software Interface de R pour les Analyses Multidimensionnelles de Textes et de Questionnaires (IRAMUTEQ) para a nuvem de palavras e método de Classificação Hierárquica Descendente $(\mathrm{CHD})^{(6)}$. Por meio do software é possível processar os dados, assim, as classes apresentadas nesse estudo foram selecionadas mediante a frequência das palavras que mais apareceram nas entrevistas fornecidas pelo programa cabendo ao pesquisador a interpretação dos dados.

\section{Procedimentos éticos}

Essa pesquisa foi aprovada pelo Comitê de Ética em Pesquisa da Faculdade de Saúde da Universidade de Brasília (CAAE 61982016.6.000.0030). Todos os envolvidos no estudo registraram a sua anuência por meio do Termo de Consentimento Livre e Esclarecido( ${ }^{(7)}$.

\section{RESULTADOS}

Pelo método de nuvem de palavras, que agrupa as palavras e as organiza graficamente em função da sua frequência, a palavra SER teve maior frequência (4l6 vezes), seguida da palavra GENTE (205 vezes) (Figura 1). 


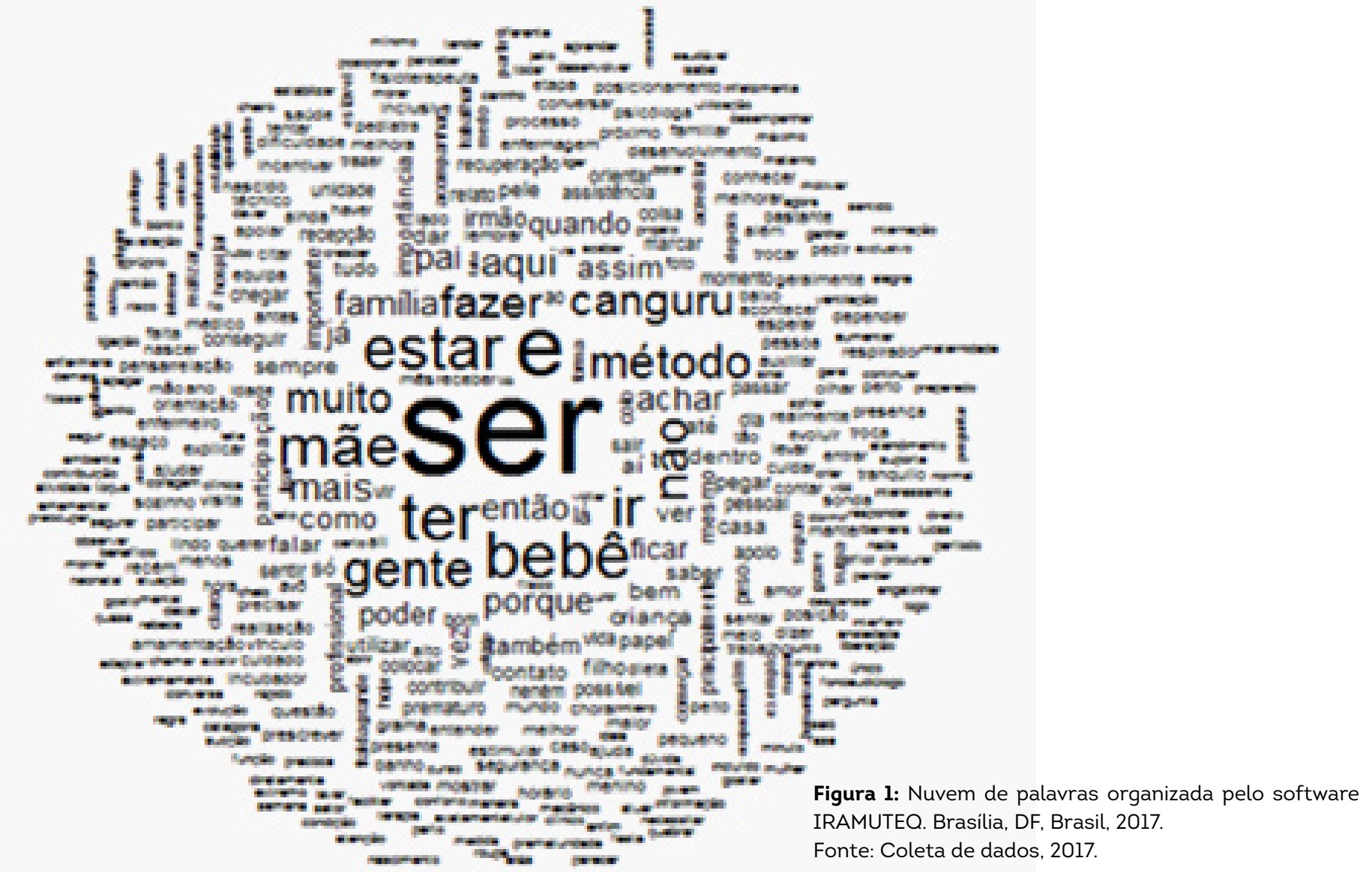

A análise de CHD resultou em um dendrograma adaptado composto por 05 classes e baseado no estudo de Almico e Faro(8), conforme Figura 2.

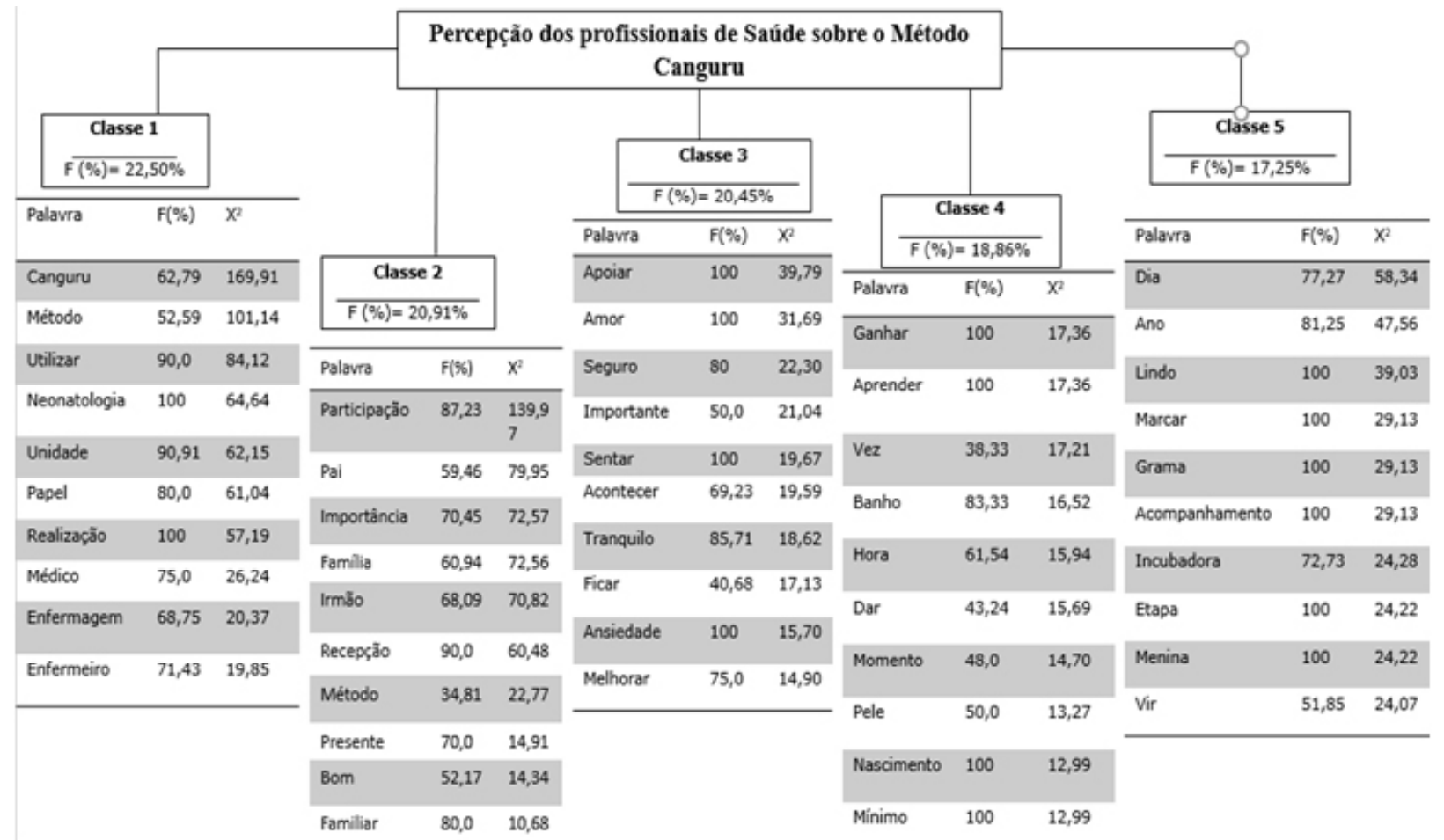

Figura 2. Dendograma e Classes Léxicas sobre a percepção dos profissionais de saúde sobre o Método Canguru. Brasília, DF, Brasil, 2017. Fonte: Coleta de dados, 2017. 
Classe 1: As atribuições de cada categoria profissional na realização do MC na unidade de neonatologia

A seguinte fala representa o conteúdo desta classe: "No método canguru todos nós somos responsáveis por todo o processo... Quando a gente fala do método canguru de trazer essa família para perto da equipe, de acolher essa família, de orientar, de proporcionar que essa família sinta que aquele bebê é dela e não de equipe isso é papel de todo mundo".(E 19)

Em outras falas foram observadas algumas particularidades de atribuições que cabem a categorias profissionais específicas: "Nós técnicos de enfermagem somos a categoria que tá mais ligado à mãe... Então assim, nós técnicos de enfermagem temos que incentivar o mais precoce possível o método canguru".(E3) "Não fica associado ao fisioterapeuta exclusivamente colocar no método canguru... Mas é importante, porque a gente fala de posicionamento, explica a importância do contato".(E8) "TO (terapeuta ocupacional) trabalha... Sempre que eu venho aqui eu dou uma olhada se os paninhos estão em cima da incubadora, se a luz tá ligada, se não tem procedimento nenhum sendo feito, o tom de voz das pessoas, se tem uma lixeira perto de uma incubadora... Eu estou sempre atuando na parte da amamentação, explicando para as mães o benefício do Método Canguru".(E9) "Eu acho que o enfermeiro ele tem que ser um incentivador... Cobrar, conversar com as mães, orientar, falar com o técnico, falar com o pai falar, com o familiar".(Ell)

\section{Classe 2: A percepção do profissional de saúde sobre a participação da família}

Uma fala que representa bem essa classe é a seguinte: "O método canguru se chamava mãe-canguru, porque se batia muito na questão da mãe, agora mudou pra método canguru... Porque o pai também tem que ser incentivado, estimulado, a fazer o método canguru e os... Irmãos aí eles não vão fazer método canguru, mas é fundamental para uma mãe que tem outros filhos saberem que o filho que tá em casa tá bem".(E17)

Embora a unidade de neonatologia proporcione a inclusão do pai no método, ainda há pouca participação dele. Todos os profissionais entrevistados se queixam deste fato e concordam que a figura paterna é essencial tanto como forma de apoio à mãe, como também para estreitar o laço que une pai e filho: "Sempre que eu posso eu coloco os bebês para fazer pele a pele com o pai, peço para o pai vir com uma camisa de botão que dê para abrir, posiciono o bebê... E eles acham um máximo, eles choram, eles arrepiam, acham a coisa mais surreal do mundo". (E9)

Alguns profissionais apoiam a participação direta ou indireta dos irmãos no MC, esse apoio também aparece nos seguinte depoimento: "Se nessa família já tem um irmão que é mais velho ele precisa ser incluido... Muitas vezes esse irmãozinho ele acaba sendo renegado a... Estar em casa, esperando uma mãe que está ausente há muitos dias, sem saber o que tá acontecendo e ele precisa ser incluído nesse processo".(E19)

Porém, observa-se em outras falas que alguns profissionais não concordam que o irmão participe do método: "Mas irmão... Tem que esperar que saia lá fora, porque a gente não pode encher muito a UTI de visita e nem trazer crianças, adolescentes ou pré-adolescentes para participar dessa nossa vida aqui dentro da UTI".(E7)

\section{Classe 3: $O$ apoio emocional à familia e amigos no ambiente extra-hospitalar}

Um discurso que simboliza esta classe é: "Se eles puderem ajudar para deixar a mãe mais tranquila aqui dentro, para ficar o máximo de tempo aqui dentro em contato com o bebê... Quando elas não têm esse apoio, elas ficam divididíssimas entre ficar aqui e apoiar lá fora".(El6)

O suporte emocional à mãe é essencial durante a permanência dela e do filho no ambiente hospitalar: "É apoiar essa mãe mesmo, porque é uma fase muito difícil, onde ela fica muito tempo fora de casa, onde ela fica muito tempo em um ambiente que ela não tá preparada para estar".(E2)

\section{Classe 4: O MC em uma unidade de neonatologia}

A fala como discurso representativo dessa classe foi: "A partir do momento que a mãe começa a pegar o bebê, sentir esse calor, ficar pele a pele a evolução dele me parece que é mais rápida".(E7)

Percebe-se que a equipe multiprofissional envolvida nos cuidados do RN identifica a melhora e evolução do quadro do RN após o contato pele a pele com a mãe e estes benefícios são expressos nas seguintes falas: "O bebê ganha peso mais rápido, ajuda na imunidade... Estimula na amamentação".(Ell) "Aumentar o vínculo da mãe com o bebê... Estabilizar questões clínicas... Estimular o afeto... Contato com o seio materno, com cheiro e o amadurecimento das funções de sucção".(El8)

A falta de profissionais em tempo integral na UCIN deixa as mães sem acompanhamento adequado para apoiálas na utilização do MC: "Mas eu acho que ainda é muito precário aqui na unidade... Acho que falta ter alguém pra incentivar, pra ficar, o espaço, a roupa".(E5)

\section{Classe 5: 0 envolvimento emocional dos profissionais de saúde na incumbência de cuidar}

Esta classe é perfeitamente representada pelo seguinte relato: "A gente vê que ele passou por tudo isso, saiu dos aparelhos, está respirando sozinho, quando ele sai daqui os pais 
trazem ele para ver o tanto que eles cresceram, progrediram, isso faz com que nós profissionais se sintam realizados, faz com que a gente sinta que a parte da gente foi feita.(El)

A equipe sente-se envolvida e feliz com a realização do seu trabalho, como na seguinte fala: "Quando a gente vê saindo de dentro da UTI para UCIN já é uma alegria... $\mathrm{Na}$ hora que a mãe vai embora ou quando a mãe traz de novo para a gente ver um bebê grande, bonitinho, todo gordinho, caminhando, é uma alegria enorme".(E7)

\section{DISCUSSÃO}

Esse estudo demonstrou que o $\mathrm{MC}$ tem sido realizado com o apoio de todos da equipe multiprofissional, respeitando sempre a estabilidade clínica do RN. Porém, destaca-se que a equipe de enfermagem encontra-se mais próxima tanto do RN quanto de seus pais.

Assim como descrito na pesquisa qualitativa realizada com 129 enfermeiras de unidades neonatais da Suécia, nesse estudo, as profissionais reconheceram que suas próprias atitudes poderiam influenciar as mães e pais, sendo assim, elas acreditam que se apoiassem a realização do MC, os familiares o realizariam ${ }^{(2)}$.

A equipe de enfermagem quanto os demais profissionais possuem função de multiplicadores de valores e práticas que podem ou não ser seguidas; desse modo, são capazes de influenciar a realização ou não do MC na prática cotidiana ${ }^{(9)}$.

Deste modo, destaca-se o papel da equipe multiprofissional como facilitadora e motivadora para a realização do MC e que podem influenciar positivamente ou negativamente a sua realização nas unidades neonatais. Cabe destacar, ainda, que os profissionais entrevistados reconhecem a pouca participação do pai e irmão na realização do MC como descrito na classe 2 .

Há uma escassez de estudos que apontam a participação do pai no método, deixando-o apenas no papel de coadjuvante ${ }^{(10)}$. Uma Revisão sistemática concluiu a existência de poucos estudos enfocando a participação do pai no MC, destacando-se como barreira para participação do pai, sua falta de oportunidade em participar ${ }^{(11)}$

A participação do pai e do irmão é fundamental como uma rede de apoio ao binômio mãe-filho; assim como apresentado na classe 3, o apoio emocional à mãe faz-se necessário durante todo o processo de internação e permanência de um RN em uma UTIN. A internação é considerada um periodo estressante e preocupante visto que influencia nas expectativas, planos e atividades cotidianas da família. Esse periodo impacta na dinâmica familiar devido à necessidade de permanência, principalmente da mãe, junto ao RN enfermo(12). Diversos sentimentos são vivenciados nesse período pelas mães como insegurança, medo, angústia e tristeza. Muitas vezes, elas se sentem culpadas, sendo o MC o meio pelo qual elas parecem redimir-se dessa culpa, ao contribuir para a alta do seu filho da UTIN, além de favorecer o amor e o vínculo afetivo(13,14). Deste modo, a mãe pode precisar de apoio de seu parceiro e familiares para praticar o $\mathrm{MC}^{(2)}$

As falas expressas na classe 4 demonstram os beneficios da realização do MC para o RN, porém destaca-se nos discursos que, apesar desses profissionais incentivarem a prática do MC, ainda existe uma lacuna para sua efetiva realização: o déficit de profissionais. Em pesquisa, já citada anteriormente, as enfermeiras também reconheceram a rotina intensa, sobrecarga de trabalho e escassez de recursos humanos como limitantes para a prática do $\mathrm{MC}^{(9)}$.

$\mathrm{Na}$ área da saúde, o déficit de recursos humanos acarreta falta de motivação para execução das funções dos profissionais, sendo a motivação um importante preditor do desempenho, eficiência, qualidade do serviço e equidade do setor de saúde ${ }^{(15)}$.

Embora o déficit de recursos humanos impacte negativamente na realização do MC, os profissionais dessa unidade sentem-se realizados e felizes por estarem envolvidos, como descrito na classe 5. As enfermeiras no Rio de Janeiro destacaram como fonte de motivação para desenvolver - MC a satisfação do profissional ao notar envolvimento, afeto e prazer de outros colegas na aplicação do método(9). Cabe ressaltar que, para a família, os profissionais de saúde quando agem de forma atenciosa, carinhosa e respeitosa, os ajudam a enfrentar a internação de forma menos traumática, além de criar um elo de confiança entre eles ${ }^{(16)}$.

\section{Limitações do estudo}

Dentre as limitações do estudo esta a ausência de participação da psicóloga, pois ela estava de licença por motivos de saúde. Além disso, as entrevistas não foram separadas de acordo com o turno do serviço (matutino, vespertino ou noturno) o que impossibilita promover um plano de ação específico por turno de trabalho.

\section{Contribuição para a prática}

As entrevistas realizadas com os profissionais que trabalham diretamente com a família e o RN internado demonstraram que o método canguru está sendo aplicado na unidade de neonatologia estudada, além de fornecerem levantamento do papel que a equipe multiprofissional desenvolve neste método. Além disso, o reconhecimento do profissional sobre a melhora e a evolução do quadro clínico do RN colocado na posição canguru contribui para que a prática do método continue sendo adotada nesta unidade, pois é uma forma de incentivo aos outros profissionais. 
A divulgação em literatura científica deste trabalho contribuirá para que a prática do método canguru seja expandida para outras unidades de neonatologia.

\section{CONCLUSÃO}

A realização desse estudo evidenciou que os profissionais da equipe multiprofissional reconhecem a importância do MC para o RN e sua família, e incentivam sua realização, porém ainda existem algumas lacunas necessárias para serem supridas como melhor adesão dos pais e irmãos ao método, e enfrentar o déficit de recursos humanos. Deste modo, sugerimos a realizações de estudos que visem promover a participação do pai e irmãos no MC.

\section{Contribuição dos autores}

A autora Josélia Rodrigues de Souza foi responsável pela realização das entrevistas, além do planejamento, execução e confecção do manuscrito. A Laiane Medeiros Ribeiro planejou, executou e confeccionou o manuscrito. Géssica Borges Vieira analisou os dados no software Iramuteq, além de contribuir na discussão e correção do manuscrito. Laíse Escalianti Del Alamo Guarda realizou a análise e interpretação dos dados, e redação do artigo. Casandra Genoveva Rosales Martins Ponce de Leon e Juliana Machado Schardosim desenvolveram a revisão crítica e revisão final do artigo.

\section{REFERÊNCIAS}

1. Phuma-Ngaiyaye E, Kalembo FW. Supporting mothers to bond with their newborn babies: Strategies used in a neonatal intensive care unit at a tertiary hospital in Malawi. International Journal of Nursing Sciences. 2016 [cited 2017 Dez 02]:3(4):362-66. Available from: http:// www.sciencedirect.com/science/article/pii/S2352013216301399

2. Mörelius E, Anderson GC. Neonatal nurses' beliefs about almost continuous parent-infant skin-to-skin contact in neonatal intensive care. Journal of clinical nursing. 2015[cited 2017 Dez 02]:24(17-18):2620-27. Available from: http://onlinelibrary.wiley.com/doi/10.1111/ jocn.12877/full

3. Soni A, Amin A, Patel DV, Fahey N, Shah N. Phatak AG, Nimbalkar SM. The presence of physician champions improved Kangaroo Mother Care in rural western India. Acta Paediatrica. 2016[cited 2017 Dez 02];105:9. Available from: http://onlinelibrary.wiley.com/doi/10.1111/ apa.13445/full

4. Boundy EO, Dastjerdi R, Spiegelman D, Fawzi WW, Missmer SA, Lieberman E, Chan GJ. Kangaroo mother care and neonatal outcomes: a meta-analysis. Pediatrics. 2015[cited 2017 Dez 02]:137(1). Available from: http://pediatrics.aappublications.org/content/pediatrics/early/2015/12/22/peds.2015-2238.full.pdf

5. Bardin L. Terceira Parte-Método. Análise de Conteúdo. Lisboa: Edições 70:93-126:1977.

6. Camargo BV, Justo AM. IRAMUTEQ: um software gratuito para análise de dados textuais. Temas em Psicologia. 2013[cited 2017 Dez 02]:21(2):513-18. Available from: http://pepsic.bvsalud.org/scielo. php?script=sci_arttextepid $=\$ 1413-389 \times 2013000200016$

7. Brasil, Ministério da Saúde. Resolução 466, Principios éticos em pesquisa com seres humanos. 12 de dezembro de 2012. Available from: http://conselho.saude.gov.br/resolucoes/2012/Reso466.pdf

8. Almico T, Faro A. Enfrentamento de cuidadores de crianças com câncer em processo de quimioterapia. Psicologia, Saúde \& Doenças. 2014[cited 2017 Dez 02];15(3),723-737. Available from: http://www.scielo.mec.pt/scielo.php?pid=S1645-00862014000300013Escript=sci arttext\&tlng=en

9. Silva LJD, Leite JL, Scochi CGS, Silva LRD, \& Silva TPD. Nurses adherence to the Kangaroo Care Method: support for nursing care management. Revista latino-americana de enfermagem. 2015[cited 2017 Dez 02]:23(3):483-490. Available from: http://www.scielo.br/ scielo.php?script=sci_arttextEpid=S0104-11692015000300483

10. Bernardo FR, Zucco LP. A centralidade do feminino no Método Canguru. Sexualidad. Salud y Sociedad-Revista Latinoamericana. 2015[cited 2017 Dez 02];21:154-174. Available from: http://www.scielo. br/scielo.php?script=sci_arttextEpid=S1984-64872015000300154

11. Seidman G, Unnikrishnan S, Kenny E, Myslinski S, Cairns-Smith S, Mulligan B, \& Engmann C. Barriers and enablers of kangaroo mother care practice: a systematic review. PloS one. 2015[cited 2017 Dez 02];10(5). Available from: http://journals.plos.org/plosone/ article?id=10.1371/journal.pone. 0125643

12. Heck GMM, Lucca HC, Costa R, Junges CF, Santos SV, \& Borck $M$. Compreensão do sentimento materno na vivência no método canguru. Revista de Enfermagem da UFSM. 2016[cited 2017 Dez 02];6(1):71-83. Available from: https://periodicos.ufsm.br/reufsm/article/view/18083

13. Nunes NP, Pessoa ÚML, Mont'Alverne DGB, Sá FED, Carvalho EM. Kangaroo care: maternal perception of the experience in the neonatal intensive care unit. Brazilian Journal in Health Promotion. 2016[cited 2017 Dez 02]:28(3):387-93. Available from: https://www.popline.org/ node/654595

14. Maia JA, Oliveira MP, Furtado SS, Silva LM, Pereira MLB. Método Canguru: a importância da familia na recuperação do recém-nascido de baixo peso. Enfermagem em Foco. 2011[cited 2018 Jan 19];2(4):231234. Available from: http://revista.portalcofen.gov.br/index.php/enfermagem/article/view/190

15. Shah SM, Zaidi S, Ahmed J, Rehman SU. Motivation and retention of physicians in primary healthcare facilities: a qualitative study from Abbottabad. Pakistan. International Journal of Health Policy and Management. 2016[cited 2017 Dez 02];5(8). Available from: https:// www.ncbi.nlm.nih.gov/pmc/articles/PMC4968250/

16. Balbino FS, Meschini GFG. Balieiro Maria MFG, Mandetta MA. Percepção do cuidado centrado na familia em unidade neonatal. Revista de Enfermagem da UFSM. 2016[cited 2017 Dez 02]:6(1):84-92. Available from: https://periodicos.ufsm.br/index.php/reufsm/article/ view/16340 